\title{
19. Chronic pain in patients with coeliac disease: Cross sectional study
}

Christianna Ioannou ${ }^{1}$, Andreas Liampas ${ }^{1, *}$, Artemios Artemiadis ${ }^{1}$, Stefania Kalampokini ${ }^{1}$, Panagiotis Bargiotas ${ }^{1}$, Georgios Hadjigeorgiou ${ }^{1}$, Panagiotis Zis ${ }^{1}$

${ }^{1}$ Medical School, University of Cyprus, Nicosia, Cyprus. * liampasand@gmail.com

Background: Coeliac disease (CD) is a long-term autoimmune disorder that primarily affects the small intestine. Classic symptoms include gastrointestinal problems such as chronic diarrhoea, abdominal distention, malabsorption, loss of appetite, and among children failure to grow normally. Often patients present with neurological manifestations, such as cerebellar ataxia and peripheral neuropathy, even in the absence of any gastrointestinal symptoms. Chronic pain is a major determinant of poor quality of life in patients with coeliac disease, however it has been previously shown that a strict gluten free diet is beneficial in dramatically reducing the odds of suffering from pain. The aim of this cross-sectional study was to establish the prevalence of chronic pain in patients with coeliac disease.

Methods: Patients with $\mathrm{CD}$ and healthy volunteers were prospectively evaluated. Pain was assessed with the use of the painDETECT and the DN4 questionnaires.

Results: Sixty-one patients with CD (79\% females, mean age $39.6 \pm 12.9$ years) and 61 age and gender matched healthy volunteers were recruited. Patients had a diagnosis of $\mathrm{CD}$ for a mean of $6.7 \pm 4.7$ years (ranging from 0 to 18 years). The prevalence of chronic pain was $57 \%$ in the healthy volunteers group and $59 \%$ in the CD group $(p=0.854)$. In both groups the prevalence of neuropathic pain was established to be $20 \%$. The most commonly reported painful area in both groups was low back pain ( $26 \%$ in the CD group and $20 \%$ in the healthy control group). In the CD group, patients with pain were significantly older compared to patients without pain ( $43.6 \pm 12.5$ years vs $33.8 \pm 11.4$ years, $p=0.003)$. No differences between these two sub-groups were found regarding gender, BMI or CD duration.

Conclusions: Chronic pain is very prevalent in $\mathrm{CD}$ and is very similar to the prevalence observed in the general population. 